\title{
Study of Design and Analysis of Low-Power 10-Transistor Full Adders Using Novel XOR-XNOR Gates
}

\author{
${ }^{1}$ Ravi Tiwari, ${ }^{2}$ Khemraj Deshmukh \\ ETC SSTC, Bhilai ,India \\ E\&I SSTC,Bhila ,India
}

\begin{abstract}
Full adders are vital components in applications such as digital signal processors (DSP) architectures and microprocessors. In this paper, we propose a technique to build a

total of 41 new 10-transistor FA using novel XOR and XNOR gates in combination with existing ones. We have done over ten thousand HSPICE simulation runs of the entire the different adders in different input patterns, frequencies, and load capacitances. Almost all those new adders consume less power in high frequencies, while 3 new adders consistently use on average 10\% less power and have higher speed compared with the previous Ten-transistor full adder and the conventional 28-T CMOS adder. One draw back of the novel adders is the threshold-voltage loss of the pass transistors.

Index Terms: Arithmetic circuit, F.A., low power, very large-scale integration (VLSI), XOR-XNOR.
\end{abstract}

\section{INTRODUCTION}

THE EXPLOSIVE growth in laptop, portable systems, and cellular networks has intensified the examine efforts in low-power microelectronics. Today, there is an ever increasing no. of portable applications requiring low power and high throughput circuits. Therefore, low-power design has become a major design consideration [2], [4], [6].

The adder is one of the most necessary components of a processor, as it is used in the automatic logic unit (ALU), in the floating-point unit, and for address generation in case of cache or memory access [2]. The full adder performance would affect the system as a whole. A variety of full adders using static or energetic logic styles have been reported in the literature [2]-[8]. In total, we have found 34 different full adder implementations, as indicated in [9]. The conventional adder uses 28 transistors implemented in CMOS technique. A new full adder called static energy-recovery full-adder (SERF) uses only 10 transistors, which has the least number of transistors and has reported to be the best in power consumption, according to [2]

Many low-power adders apply circuit level techniques using various pass transistors, such as the SERF [2]. Compared to the complementary static CMOSadders, such low-power adders have the problem of threshold loss, i.e., the logic value 1 is not the value of and the logic value 0 may not be the value of 0 vol. This kind of threshold-loss logic gates may not be used as widely as the complementary static CMOS gates. However, they are positively useful in building up larger circuits such as multiple- bit input adders and multipliers. One such established application is the Manchester carry-look ahead chain (MCC) [10] where pass transistors are used to generate carry functions and all pass transistors suffer the threshold-loss problem, yet the final adder circuit functions correctly as reported in [10].

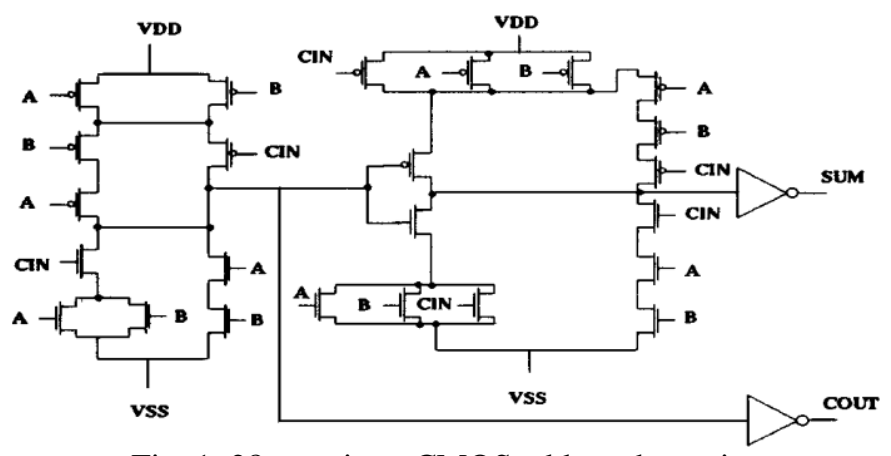

Fig. 1. 28-transistor CMOS adder schematic. 


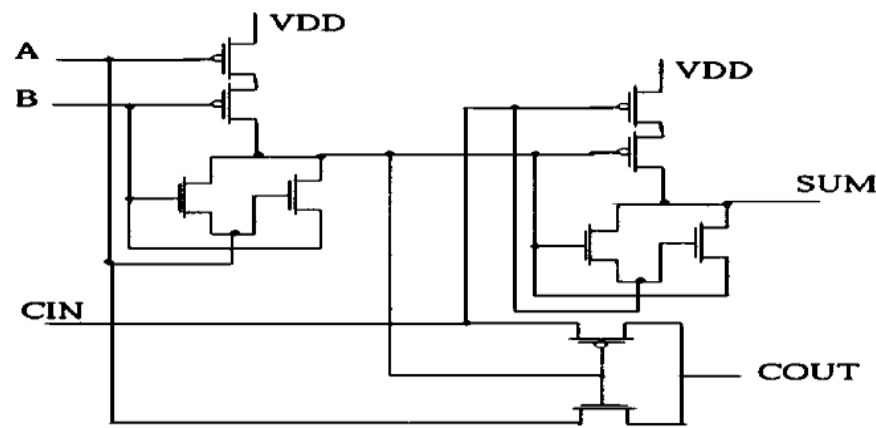

Fig. 2. SERF adder.

In this paper, we propose a systematic approach to designing many 10-transistor full adders. Our new adders also have the threshold-loss problem; however, the adders are useful in bigger circuits such as multipliers despite the threshold-loss problem. Using a novel set of XOR-XNOR gates in combination

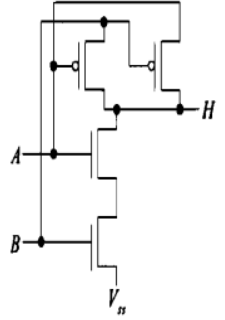

$X O R$

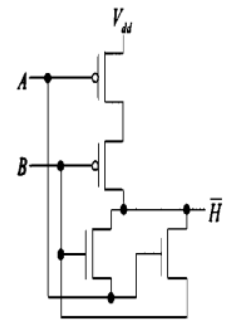

XNOR

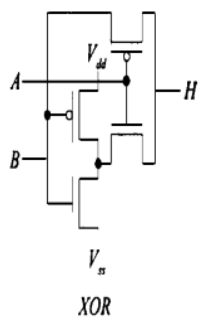

XOR

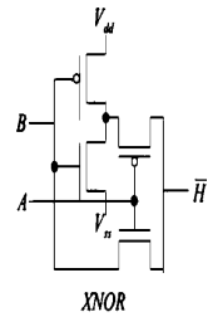

XNOR

with existing ones, a total of 41 new 1-bit full-adders are created. We have conducted over 10000 HSPICE simulation runs of all the different adders in dissimilar input patterns, frequencies, and loading conditions. The reason to do these many simulations is to give a better confidence to how the new adders would perform under all the possible practical applications. Of course we can never cover all the possible practical cases, since no one can do so due to the fast change in the circuit and technology areas. Almost all those new adders show lowpower consumption in high frequencies. In particular, three new adders consistently consume on average $10 \%$ less power and have higher speed compared with the previous 10-transistor full adder and the conventional 28transistor CMOS adder.

The rest of the paper is organized as follows: In Section II we briefly describe the previous work in literature. In Section III we propose the new adders. In Section IV we present the simulation methodology and simulation results. In Section V, we draw the conclusions.

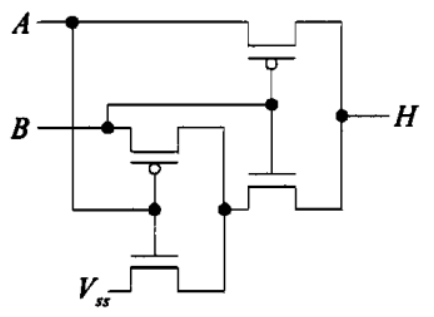

$X O R$

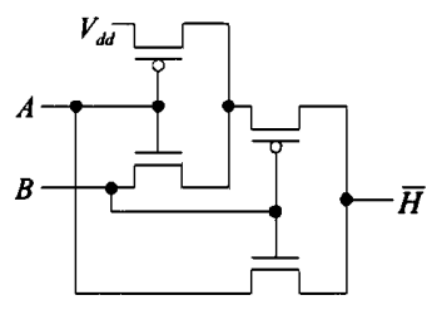

XNOR

\section{Previous Work}

The addition of $2 \mathrm{~A}$ and B with Cin yields a SUM and A Cout bit. The integer equivalent of this relation is shown by the (2) and (3) or (4)-(6) 
TABLE I

DESCRIPTIONS OF ALL ADDERS

\begin{tabular}{|c|c|c|c|c|c|}
\hline NAME & MODULE 1 & IN1 & IN2 & MODULE 2 & COUT \\
\hline $1 \mathrm{~A}$ & XOR & MID & CIN & $\mathrm{XOR}$ & MUXX \\
\hline 18 & XOR & CIN & MID & XOR & MUIX \\
\hline $1 C$ & XOR & CIN & MID & $\mathrm{XOR}$ & PMOS: \\
\hline $1 D$ & INV XOR & SINL & MID & XOR & NMOS \\
\hline $2 A$ & $P-A G-X N N O R$ & MID & CIN & XNOR & MUX \\
\hline $2 \mathrm{~B}$ & $P-K t=X N O R$ & SIN & MID & $\mathrm{P}-\mathrm{AG}-$ & MUX \\
\hline $3 A$ & $P-K G-\quad X O R$ & MID & CIN & $P-N G$ - & MUX \\
\hline $3 B$ & $R-K \mathbf{S}_{-}$ & CIN & $M \Pi D$ & P- $-1 G-$ & MUUX \\
\hline $4 A$ & INV XNOR & CIN & MID & XNOR & MUX \\
\hline $4 B$ & INV XNOR & MID & CIN & XINOR & MUIX \\
\hline $4 C$ & $\mathrm{XNOR}$ & $M$ & CIN & $\mathrm{XNOR}$ & PMOS \\
\hline $4 D$ & XNOR & MID & CIN & $\mathrm{XNOB}$ & NMOS \\
\hline SA & XNOR & MID & CIN & XNOR & MUX \\
\hline SB & INV XINOR & CIN & MID & $P-1 G=$ & MUX. \\
\hline 6A. & $P-N G-X N O R$ & CIN & MID & XNNQR & MUX \\
\hline $6 \mathrm{~B}$ & $P-K t-X I N O R$ & MID & CIN & XNOR & MUX \\
\hline 6C & $P-N G-X N O R$ & MID & CIN & $\mathrm{XNOB}$ & PMOS \\
\hline 6D & $P-N G-X N O R$ & MID & CIN & XNOB & NMOSS \\
\hline $7 \mathrm{~A}$ & INV XOR & MID & CIN & $\mathrm{P}-k \mathrm{G}-$ & MUX \\
\hline $7 \mathbf{B}$ & $\mathrm{XOR}$ & CIN & MID & $\mathrm{P}-\mathrm{NG}-$ & MuX \\
\hline $8 A$ & $P-K G=X O R$ & MID & CIN & $\mathrm{XOR}$ & MUX \\
\hline 8B & $P-N G-$ & CIN & MID & XOR & MUX \\
\hline $8 c$ & $P-K G=$ & CIN & MID & XOB & PMOS \\
\hline 8D & $P-N G-X O R$ & CIN & MID & $\mathrm{XOR}$ & NMAOS \\
\hline 9A & SER XNOR & MID & CIN & $\mathrm{P}-\mathrm{NG}-$ & MUX \\
\hline 98 & SER XNOR & CIN & MID & $P-N G=$ & MuX \\
\hline $10 \mathrm{~A}$ & $P-N G-X N O R$ & MID & CIN & XNOR & MUX \\
\hline $11 \mathrm{~A}$ & SER XINR & MID & CIN & $P-N C=$ & MUX \\
\hline $11 \mathrm{~B}$ & SER XINOR & CIN & MID & $\mathrm{P}-\mathrm{NG}=$ & MUX \\
\hline $12 A$ & $P-N G-X O R$ & MID & CIN & XOR & MUX \\
\hline $13 \mathrm{~A}$ & SER XNORR & SIN & MID & XNOR & MUX \\
\hline $13 \mathrm{~B}$ & XNOR & MID & CIN & $\mathrm{XNOR}$ & MUX \\
\hline $13 c$ & XNOR & MID & SIN & XNOR & PMOS \\
\hline $13 D$ & SER XNOOR & MID & CIN & XNOR & NMOS \\
\hline $14 A$ & INV XNOR & MID & SIN & $\mathrm{XNOR}$ & MUX \\
\hline $15 \mathrm{~A}$ & $\mathrm{XOR}$ & MID & CIN & XOR & MUIX \\
\hline $15 \mathrm{~B}$ & $\mathrm{XOR}$ & CIN & MID & $\mathrm{XOR}$ & MUX \\
\hline $15 \mathrm{C}$ & $\mathrm{XOR}$ & CIN & MID & XOR & PMOS \\
\hline $15 \mathrm{D}$ & $\mathrm{XOR}$ & CIN & MID & $\mathrm{XOR}$ & NMOS \\
\hline $16 \mathrm{~A}$ & $\mathrm{XOR}$ & MID & CIN & $\mathrm{XOB}$ & MUX \\
\hline $17 A$ & $\mathrm{XOR}$ & MID & CIN & $\mathrm{XQR}$ & MUX \\
\hline $18 \mathrm{~A}$ & SER XNOR & MID & CIN & $\mathrm{XNOR}$ & MUX \\
\hline
\end{tabular}

$\mathrm{A}+\mathrm{B}+\mathrm{Cin}=2 *$ Cout $+\mathrm{SUM}$

Cout $=(\mathrm{A} \wedge \mathrm{B}) \vee((\mathrm{A} \vee \mathrm{B}) \wedge \mathrm{Cin})$

$\mathrm{SUM}=(\mathrm{A} \wedge \mathrm{B} \wedge \mathrm{Cin}) \vee(\mathrm{A} \vee \mathrm{B} \vee \mathrm{Cin}) \wedge($ Cout' $)$

$\mathrm{SUM}=\mathrm{A}$ exor $\mathrm{B}$ exor $\mathrm{Cin}$

$\mathrm{SUM}=\mathrm{A}$ exnor $\mathrm{B}$ exnor Cin

Cout $=(A \wedge(A$ exnor $B)) \vee(C$ in $\wedge(A$ exor $B))$

Many full adder designs can be found in the literature. In [3], a 28-transistor adder shown in Fig. 1 implements (2) and (3) using complementary CMOS design. The SERF adder, shown in Fig. 2, implements (5) and (6) using only ten transistors.

Our newly proposed adders also implement (4) or (5) and (6), using XOR-XNOR gates as the basic building blocks. Several designs of XOR-XNOR have been proposed in the literature. At the time of this publication, the smallest XOR-XNOR gates have four transistors. Two of such XOR-XNOR gates are shown in Fig. 3(a) and (b) [1].

\section{a. Novel XOR and XNOR Gates}

\section{EW DESIGN}

Before we present the new adders, we first propose a new XOR gate, shown in Fig. 4. It resembles the inverterbased XOR shown in Fig. 3(b) but the difference is that the VDD connection in the inverter-based XOR is connected to the input A. Because the new XOR gate has no power supply, it is called Powerless 


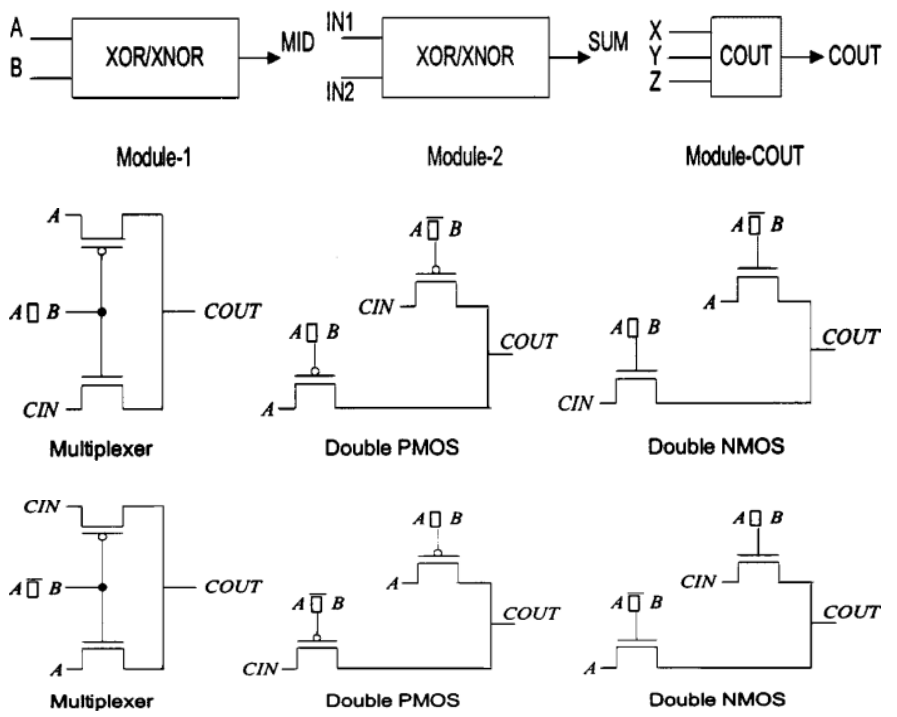

Fig. 6. Choices for module-COUT. (a) Module-COUT for module- $1=$ module-2 $=$ XOR. (b) Module-COUT for module- $1=$ module- $2=$ XNOR.

\section{B. Full Adder}

We use three modules, shown in Fig. 5, to implement the full adder based on (4) or (5) and (6). Module-1 and module-2 can be XOR or XNOR gates and module- can be a multiplexer, double PMOS or double NMOS transistors. The sum is generated by cascading module- 1 and module- 2 . This implements (4) or (5). The function is implemented by module-1 and module- according to (6). If module- 1 and module- 2 are XOR gates, module- can have one of the configurations shown in Fig. 6(a). If module-1 and module-2 are XNOR gates, the choices for the moduleare shown in Fig. 6(b). The first 18 full adders use the multiplexer as module- Modules 1 and 2 either have to be both XOR or both XNOR. They can be inverter-based XOR-XNOR, static energy-recovery XOR-XNOR or P-/G-XOR-XNOR. For example, with an inverter-based XOR module1 , module-2 can be inverter-based XOR, static energy-recovery XOR or P-/G-XOR. There are three possible choices for module-1 and three possible choices for module-2. This gives nine combinations for the case when modules 1 and 2 are XOR and nine more when they are XNOR. They are referred to as the initial adder subset shown in Fig. 7. They are also listed in Table I as all the adders whose names end with the letter "A" (1A-18A). A new adder subset, called the wire switch, is derived from the initial adder subset by exchanging the two inputs to module-2. An adder from the wire switch adder subset has the

\begin{tabular}{|c|c|c|c|c|c|c|c|}
\hline \multicolumn{8}{|c|}{ MODULE-COUT } \\
\hline \multicolumn{2}{|c|}{$\begin{array}{l}\text { MULTIPLEXOR } \\
\text { (INITIAL) }\end{array}$} & \multicolumn{2}{|c|}{$\begin{array}{l}\text { MULTIPLEXOR } \\
\text { (WIRE SWITCH) }\end{array}$} & \multicolumn{2}{|c|}{ NMOS } & \multicolumn{2}{|c|}{ PMOS } \\
\hline Module-1 & Module-2 & Module- & Module-2 & Module- & Module-2 & Module- & Module-2 \\
\hline INV & INV & INV & INV & INV & INV & INV & INV \\
\hline INV & SER & & & & & & \\
\hline INV & $P-/ G-$ & INV & $P-K G-$ & & & & \\
\hline SER & INV & SER & INV & SER & INV & SER & INV \\
\hline SER & SER & & & & & & \\
\hline SER & $\mathrm{P}-/ \mathrm{G}-$ & SER & $P-/ G-$ & & & & \\
\hline$P-/ G-$ & INV & $P-N G-$ & INV & $P-/ G-$ & INV & $P-/ G-$ & INV \\
\hline$P-/ G-$ & SER & & & & & & \\
\hline$P-/ G-$ & $P-/ G-$ & $P-/ G-$ & $P-/ G-$ & & & & \\
\hline
\end{tabular}

Fig. 7. Adder variations.

same three modules as its corresponding adder from the initial adder subset. Each initial adder produces a wire switch adder. There are six cases when module-2 is a static energy-recovery XOR-XNOR (Fig. 7). Static energy-recovery XOR-XNOR gates are symmetric and thus, a wiring change does not alter their performance. These six adders are, therefore, identical to their initial adder subset counterpart and are not counted. The wire switch adder subset consists of 12 adders (18 minus 6 that have static energy-recovery XOR-XNOR). The wire switch adders are shown in Fig. 7 and are listed in Table I as adders whose names end with the letter "B." Using the wire switch adder subset, we can obtain more variations by replacing module Cout- while keeping modules1 and 2. Module Cout- is replaced by one of the choices shown in Fig. 6. These choices for module- Cout require both A exor B and A exnor B. With the available XOR-XNOR gates, the only way is to have an inverter-based $\mathrm{XOR}-\mathrm{XNOR}$ as module-2. The first signal to module- Cout is taken from the output of 


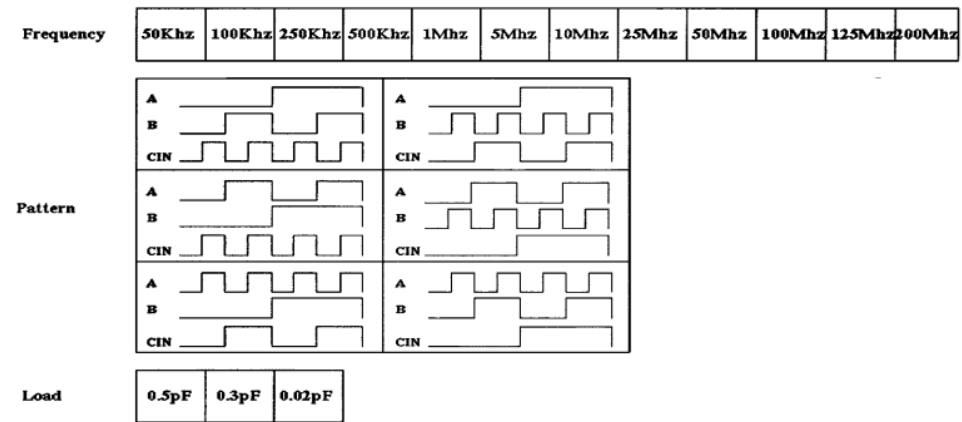

Fig. 8. Simulation setups: input patterns and loading conditions.

SERF
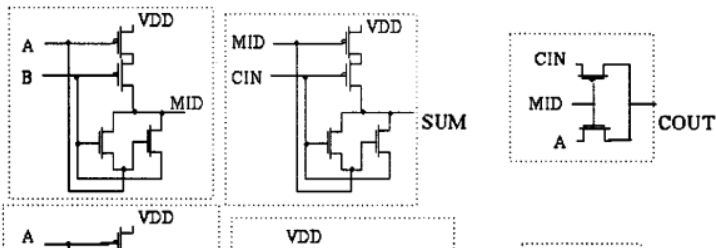

ADDER 9A
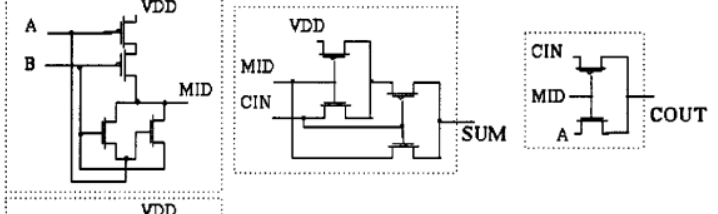

ADDER 9B
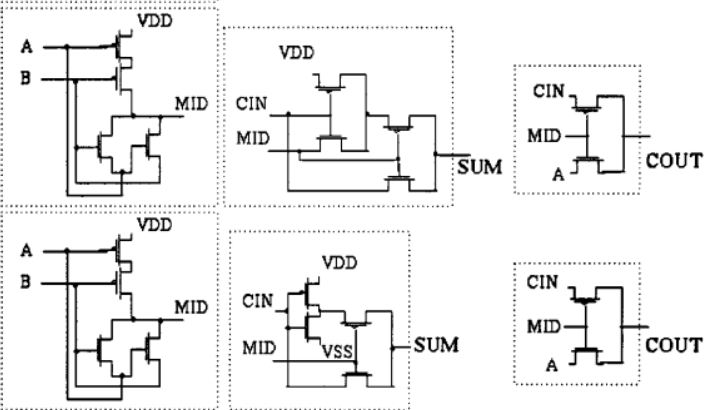

ADDER 13A
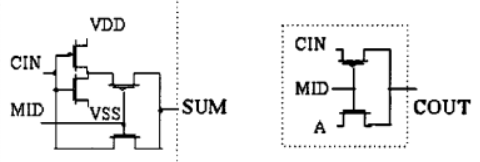

Fig. 9. Construction modules to build various adders.

module-1 and its complement is taken after the CMOS inverter of module-2. There are six adders that use the inverter-based XOR-XNOR as module-2. Thus, there are six new adders that use the double PMOS and six more that use the double NMOS as their module-Cout. They are listed in Table I as adders whose names end with the letter " $\mathrm{C}$ " for PMOS and " $\mathrm{D}$ " for NMOS.

The above process creates a total of 42 adders which are listed in Table I. One of the adders generated through this process is the SERF adder, listed as 18A. Hence, there are 41 different new full adders. The columns IN1 and IN2 in Table I show which wires are connected to the two inputs of module-2. The Cout column shows the selected module- Cout.

\section{Experiment Description And The Results}

We have performed experiments on the 41 newly designed 1-bit full adders along with the SERF adder and the conventional CMOS adder at the schematic level. The transistors have a channel length of $0.6 \mathrm{~m}$ and a channel width of $2.4 \mathrm{~m}$ using $3.3 \mathrm{~V}$ logic. Each circuit is simulated with the same testing conditions. The netlists of those adders are extracted and simulated using HSPICE on an Ultra-SPARC 2 machine.

A circuit responds differently to different input patterns. So we use six input patterns to cover all input combinations. Those patterns are shown in Fig. 8. Each pattern is simulated 12 times using frequencies ranging from $50 \mathrm{kHz}$ to $200 \mathrm{MHz}$. Three different capacitor values $(0.5,0.3$, and $0.02 \mathrm{pF})$ are used to load the outputs. 

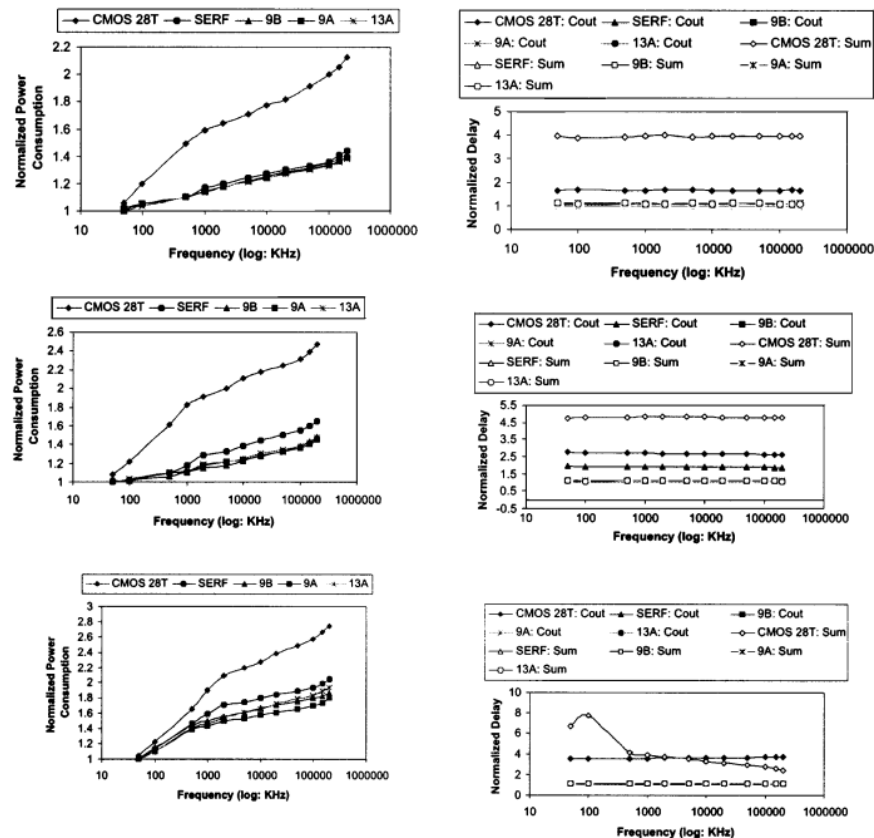

Fig. 10. Comparison of power consumption and critical delay.

Thus, for each adder, 216 HSPICE simulation runs ( 6 patterns $* 12$ frequencies $* 3$ loads) are made. This gives a total of 9288 simulation runs for the preliminary part of the experiment (43 adders $* 216$ simulations/adder). The simulation setup is shown in Fig. 8. For every pattern, frequency and load combination, we have 1 SUM power measurement, 1 Cout delay measurement and 1 delay measurement. For a given load, input pattern and frequency, four complete pattern cycles are simulated. All input signals have a rise time and a fall time of 500 ps. This is a suitable arrangement for the adopted technology in this study. For finer technologies, we shall expect lower rise and fall times. The simulation results shall be very similar to what is reported to our simulation runs. During a simulation session, a single power measurement is taken by averaging the instantaneous power over a period of three pattern cycles starting from the beginning of the second cycle to the end of the fourth cycle. The measurement does not include the first cycle to avoid transient glitches. The average power of an adder is the average value of the six different pattern power measurements. Thus, for a specific load, an adder has 12 different average power measurements, accounting for each of the 12 input frequencies.

Propagation delay is the time between the fastest input signal and the output signal. We use the first rising edge of all signals at the beginning of the second pattern cycle. The critical propagation delay is the value of the highest delay measured for the Cout and SUM output for the six different patterns with a specific load and frequency.

After the simulations, three of the new full adders stand out as being the best: adder 9A, adder 9B, and adder 13A, shown in Fig. 9. Along with the SERF adder and the CMOS adder (Fig. 1), these adders have been simulated under 11 new loading conditions varying from 0.01 to $0.5 \mathrm{pF}$. Each load simulation is done with six different patterns at 12 different frequencies. This gives an additional 3960 simulation runs (72 simulations/load $* 11$ loads/adder * 5 adders), in addition to the previous 9288 simulations. In total, there are 13248 simulation runs.

In power consumption, adder 9B consistently has better power consumption than the SERF adder. It consumes up to $12 \%$ less power. Adder $9 \mathrm{~A}$ and adder 13A have better power consumption except when the load is $0.01 \mathrm{pF}$. Adder $9 \mathrm{~A}$ consumes up to $20 \%$ less power whereas adder $13 \mathrm{~A}$ consumes up to $10 \%$ less.

The CMOS adder dissipates more power than the other adders. The left-hand side of Fig. 10 shows the power measurement for loads of $0.01,0.25$, and $0.5 \mathrm{pF}$, respectively, starting from the top.

Adders 13A and 9B have better critical delay than the SERF adder in all loading condition. They have up to 93\% better speed than the SERF adder. The right-hand side of Fig. 10 shows the propagation delay at key loading conditions: $0.02 \mathrm{pF}$ at the top, $0.15 \mathrm{pF}$ at the middle, and $0.45 \mathrm{pF}$ at the bottom.

As mentioned earlier, the performance of many larger circuits are strongly dependent on the performance of the full adder circuits that have been used. The new 10-transistor adder circuits presented in this study, are good candidates to build these large systems, such as high performance multipliers with low power consumption. The small area of these adders can also significantly reduce the area of the systems built upon them. 


\section{Conclusions}

In this paper, we have presented a systematic approach to construct full adders using only ten transistors. In total we can construct 42 ten-transistor adders of which 41 are new. Based on our extensive simulations, we conclude that three new adders consume on average 10\% less power and have $90 \%$ higher speed compared to the previous ten-transistor adder.

\section{References}

[1] J. Wang, S. Fang, and W. Feng, "New efficient designs for XOR and XNOR functions on the transistor level," IEEE J. Solid-State Circuits, vol. 29, pp. 780-786, July 1994.

[2] R. Shalem, E. John, and L. K. John, "A novel low power energy recovery full adder cell," in Proc. IEEE Great Lakes VLSI Symp., Feb. 1999, pp. 380-383.

[3] N.Weste and K. Eshraghian, Principles of CMOSVLSI Design, A System Perspective. Reading, MA: Addison-Wesley, 1993.

[4] A. P. Chandrakasan, S. Sheng, and R. W. Brodersen, "Low-power CMOS digital design," IEEE J. Solid-State Circuits, vol. 27, pp. 473-483, Apr. 1992.

[5] H. T. Bui, A. K. Al-Sheraidah, and Y.Wang, "New 4-transistor XOR and XNOR designs," Tech. Rep., Florida Atlantic Univ., Boca Raton, 1999.

[6] R. Pedram and M. Pedram, Low Power Design Methodologies. Norwell, MA: Kluwer, 1996

[7] R. Zimmermann and W. Fichtner, "Low-power logic styles: CMOS versus pass-transistor logic," IEEE J. Solid-State Circuits, vol. 32, pp. 1079-1090, July 1997.

[8] T. Callaway and E. Swartzlander, Jr., "Low power arithmetic components," in Low Power Design Methodologies. Norwell, MA: Kluwer, 1996, pp. 161-201.

[9] Y. Wang, Y. Jiang, and J. Wu, “Comprehensive power evaluation of full adders,” Tech. Rep., Florida Atlantic Univ., Boca Raton, 2000 .

[10] T. Lynch and E. Swartzlander, “A spanning tree carry lookahead adder,” IEEE Trans. Comput., vol. 41, pp. 931-939, Aug. 1992. 